\title{
Stability Analysis of Nonlinear Systems using Frozen Stationary Linearization
}

\author{
Anders Hansson, Anders Helmersson, Torkel Glad \\ Division of Automatic Control \\ Department of Electrical Engineering \\ Linköpings universitet, SE-581 83 Linköping, Sweden \\ WWW: http://www. control.isy.liu.se \\ E-mail: hansson@isy.liu.se, andersh@isy.liu.se \\ torkel@isy.liu.se
}

2nd April 2003

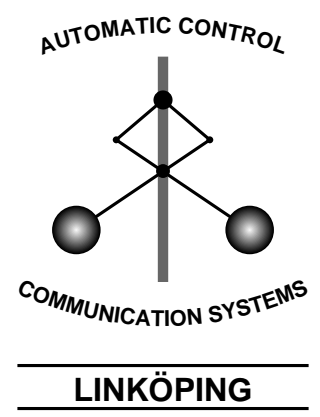

Report no.: LiTH-ISY-R-2512

Submitted to IEEE CDC 2003

Technical reports from the Control \& Communication group in Linköping are available at http://www. control.isy.liu.se/publications. 


\begin{abstract}
In this paper is discussed how to compute stability regions for nonlinear systems with slowly varying parameters using frozen stationary linearization. It is shown that larger stability regions can be obtained as compared to traditional approaches using recent stability results for linear parameter-varying systems.
\end{abstract}

Keywords: Nonlinear Systems, Stability Analysis, Lyapunov Stability, Linear Parameter-Varying Systems, Gain-Scheduling 


\title{
Stability Analysis of Nonlinear Systems using Frozen Stationary Linearization
}

\author{
Anders Hansson, Anders Helmersson and Torkel Glad \\ Division of Automatic Control \\ Department of Electrical Engineering \\ Linköping University \\ SE-581 83 Linköping, Sweden \\ hansson@isy.liu.se
}

\begin{abstract}
In this paper is discussed how to compute stability regions for nonlinear systems with slowly varying parameters using frozen stationary linearization. It is shown that larger stability regions can be obtained as compared to traditional approaches using recent stability results for linear parametervarying systems.
\end{abstract}

Key Words: Nonlinear Systems, Stability Analysis, Lyapunov Stability, Linear Parameter-Varying Systems, GainScheduling.

\section{Introduction}

Stability analysis of nonlinear differential equations has been an active research area since the pioneering work by Lyapunov in 1892, e.g. [11]. Local stability analysis based on linearization around a stationary point is taught in undergraduate courses. Linearization around a nominal trajectory and optimal control of the resulting time-varying system was introduced in the early optimal control litterature, e.g. [5, Chapter 6.4], and [4]. The derivation of the time-varying linear system is in general quite involved, since it is required to find the nominal solution. Especially this is the case when investigating input-to-state stability. For slowly varying systems this problem can be circumvented by considering the input as a "frozen" parameter. This has important applications in design and analysis of gain-scheduled controllers.

Gain-scheduling is a very powerful control methodology for control of systems with varying process dynamics that can be predicted. It was originally used for flight control systems, [19]. With digital implementations it s now increasingly used also in process control. Other areas where gain scheduling is applied are e.g. autopilots for ships and combustion control for cars, [2]. Scheduling variables in flight control systems are typically velocity, altitude and angle of attack. In process industry a typical scheduling variable is production rate.
Traditionally, the design of gain-scheduled controllers is carried out by first linearizing the system to be controlled at a discrete number of operational points parameterized by the scheduling variables. Then linear controllers are designed for each of these operational points, and the overall controller is obtained by interpolation. Even if each linear controller is stable for its linearized system model, there is no guarantee that the overall control scheme is stable when the parameter that the scheduling is based on starts to vary, [18]. However, if bounds on the rate of variation of the parameter is imposed it is possible to show that stability is recovered, $[10,16,9,17,15]$. Common to these approaches is that they all assume that the linearized system is exponentially stable, uniformly in the parameter. Under a rate-constraint on the parameter they then typically show that the nonlinear system is uniformly ultimately bounded locally. Recently much attention has been given to analyzing the Linear Parameter Varying (LPV) system, i.e. the linearized system for time-varying parameters, e.g. $[6,1,7]$. It has been shown that computationally attractive schemes can be derived to construct parameter-dependent Lyapunov-functions which prove global stability for the LPV system. It is the scope of this paper to make of use of these new results to obtain larger stability regions for the nonlinear system.

The remaining part of the paper is organized as follows. In Section 2 the nonlinear system is defined together with the "frozen" stationary points. Also the linearized system is derived. In Section 3 the stability result is presented. It is shown that the solution is stable assuming that the parameters vary slowly. In Section 4 the results of this paper are related to previous work. In Section 5 it is discussed how to compute Lyapunov functions for the LPV system. In Section 6 an example is investigated, and it is shown that the stability region is larger for the proposed approach as compared to previous approaches. Finally, in Section 7 some conclusions and remarks about extensions are given. 


\section{Preliminaries}

Consider the nonlinear differential equation

$$
\dot{x}=f(x, \rho), \quad \forall t \geq 0
$$

where $x(t) \in \mathbf{R}^{n}$ denotes the state vector, and where $\rho(t) \in$ $\Gamma \subset \mathbf{R}^{m}, \forall t \geq 0$ is a parameter vector. We remark that any explicit time-dependence in $f$ can be taken care of by incorporating $t$ as a component of $\rho$. When analyzing the system defined by (1) we linearize around a "frozen"stationary point, [8, Chapter 5.7], i.e. $x_{0}$ should satisfy

$$
f\left(x_{0}(t), \rho(t)\right)=0, \quad \forall t \geq 0
$$

Notice that we can express $x_{0}(t)$ as a relation with $\rho(t)$, which will only depend on the current time $t$. This is a standard approach for slowly varying parameters $\rho$. We will assume that (2) defines $x_{0}(t)$ as a function of $\rho(t)$, i.e. $x_{0}(t)=\varphi(\rho(t))$. We will also assume that $\varphi$ is differentiable with respect to $\rho$. The analysis will be based on the linearized system

$$
\dot{z}=A(\rho) z
$$

where $A(\rho)=\frac{\partial f}{\partial x}(\varphi(\rho), \rho)$. This system is what is called a LPV system.

Define the change of variables $z=x-x_{0}$. This will transform the system into

$$
\dot{z}=g(z, \rho)-\dot{x}_{0}
$$

where $g(z, \rho)=f(z+\varphi(\rho), \rho)$. Notice that

$$
\dot{x}_{0}=\frac{\partial \varphi}{\partial \rho}(\rho) \dot{\rho}
$$

We assume that

$$
\left\|\frac{\partial \varphi}{\partial \rho}\right\|_{2} \leq \beta
$$

$\forall \rho \in \Gamma$. In case we know that certain parameters do not vary with time we may remove the corresponding components in $\dot{\rho}$ and the corresponding columns in $\frac{\partial \varphi}{\partial \rho}$ in the expression for $\dot{x}_{0}$. This may result in lower values of $\beta$. In what follows we will assume that $g: D \times \Gamma \rightarrow \mathbf{R}^{n}$ is such that solutions of (4) are well defined $\forall t \geq 0, \forall \rho \in \Gamma$. We will also assume that the Jacobian matrix $\partial g / \partial z$ is bounded and Lipschitz on $D$, uniformly in $t$ and $\rho$, i.e.

$$
\left\|\frac{\partial g_{i}}{\partial z}\left(z_{1}, \rho\right)-\frac{\partial g_{i}}{\partial z}\left(z_{2}, \rho\right)\right\|_{2} \leq L_{1}\left\|z_{1}-z_{2}\right\|_{2}
$$

$\forall z_{1}, z_{2} \in D, \forall \rho \in \Gamma$.

\section{Stability Analysis}

We will now investigate stability of solutions $x$ of (1) or equivalently solutions $z$ of (4). We will show stability assuming that the LPV system admits a global Lyapunov function. We have to require that $\left\|\dot{x}_{0}(t)\right\|_{2} \leq \gamma, \forall t \geq 0, \forall \rho \in \Gamma$, where $\gamma$ is some constant. The stability concept for the nonlinear system is that if $z(0) \in E \subset D$, then $z(t)$ will converge to a region $F \subset E$ in finite time. Hence the solution of (4) is uniformly ultimately bounded, e.g. [8, Definition 5.1].

By the mean value theorem

$$
g_{i}(z, \rho)=g_{i}(0, \rho)+\frac{\partial g}{\partial z}\left(\zeta_{i}, \rho\right) z
$$

where $\zeta_{i}$ is a point on the line segment connecting $z$ to the origin. Since $g_{i}(0, \rho)=0$ by construction, we have

$$
\begin{aligned}
g_{i}(z, \rho) & =\frac{\partial g}{\partial z}\left(\zeta_{i}, \rho\right) z=\frac{\partial g}{\partial z}(0, \rho) z \\
& +\left[\frac{\partial g}{\partial z}\left(\zeta_{i}, \rho\right)-\frac{\partial g}{\partial z}(0, \rho)\right] z
\end{aligned}
$$

Hence

$$
g(z, \rho)=A(\rho) z+h(z, \rho)
$$

where

$$
h_{i}(z, \rho)=\left[\frac{\partial g}{\partial z}\left(\zeta_{i}, \rho\right)-\frac{\partial g}{\partial z}(0, \rho)\right] z
$$

The function $h(z, \rho)$ satisfies

$$
\|h(z, \rho)\|_{2} \leq L\|z\|_{2}^{2}
$$

where $L=\sqrt{n} L_{1}$ by (7). Now assume that there is a continuous, differentiable, bounded, positive definite, symmetric matrix $P(\rho)$ such that

$$
0<c_{1} I \leq P(\rho) \leq c_{2} I
$$

and

$$
\dot{P}(\rho)+P(\rho) A(\rho)+A^{T}(\rho) P(\rho) \leq-c_{3} I<0
$$

$\forall t \geq 0, \forall \rho \in \Gamma$, where $\dot{P}(\rho)=\sum_{i=1}^{m} \frac{\partial P}{\partial \rho_{i}}(\rho) \dot{\rho}_{i}$. We remark that it is trivial to show that this is a global Lyapunov function for the LPV system in (3). We will now use $V(z, \rho)=z^{T} P(\rho) z$ as a Lyapunov function candidate for the nonlinear system (4). The derivative of $V(z, \rho)$ along the trajectories of the system is given by

$$
\begin{aligned}
\dot{V}(z, \rho) & =z^{T} P(\rho)\left[g(z, \rho)-\dot{x}_{0}\right] \\
& +\left[g(z, \rho)-\dot{x}_{0}\right]^{T} P(\rho) z+z^{T} \dot{P}(\rho) z \\
& =z^{T}\left[P(\rho) A(\rho)+A^{T}(\rho) P(\rho)+\dot{P}(\rho)\right] z \\
& +2 z^{T} P(\rho)\left[h(z, \rho)-\dot{x}_{0}\right] \\
& \leq-c_{3}\|z\|_{2}^{2}+2 z^{T} P(\rho)\left[h(z, \rho)-\dot{x}_{0}\right] \\
& \leq-c_{3}\|z\|_{2}^{2}+2 c_{2} L\|z\|_{2}^{3}+2 c_{2} \gamma\|z\|_{2} \\
& =\left[-c_{3}+2 c_{2}\left(L\|z\|_{2}+\frac{\gamma}{\|z\|_{2}}\right)\right]\|z\|_{2}^{2}
\end{aligned}
$$

if $\left\|\dot{x}_{0}(t)\right\|_{2} \leq \gamma, \forall t \geq 0, \forall \rho \in \Gamma$.

The region $E \backslash F$ in which $\dot{V}(z, \rho)<0$ is given by the set of $z$ such that $z \in D$ and

$$
-c_{3}+2 c_{2}\left(L\|z\|_{2}+\frac{\gamma}{\|z\|_{2}}\right)<0
$$


which is equivalent to

$$
\left(\|z\|_{2}-\frac{c_{3}}{4 c_{2} L}\right)^{2}-\frac{c_{3}^{2}}{16 c_{2}^{2} L^{2}}+\frac{\gamma}{L}<0
$$

We first notice that there is a solution to this inequality if and only if

$$
\gamma<\frac{c_{3}^{2}}{16 c_{2}^{2} L}
$$

The sets $E$ and $F$ are hence given by

$$
\begin{aligned}
& E=\left\{z \mid\|z\|_{2} \leq \frac{c_{3}}{4 c_{2} L}+\sqrt{\frac{c_{3}^{2}}{16 c_{2}^{2} L^{2}}-\frac{\gamma}{L}}\right\} \cap D \\
& F=\left\{z \mid\|z\|_{2} \leq \frac{c_{3}}{4 c_{2} L}-\sqrt{\frac{c_{3}^{2}}{16 c_{2}^{2} L^{2}}-\frac{\gamma}{L}}\right\} \cap D
\end{aligned}
$$

Notice that because of (6) we may replace the condition on $\dot{x}_{0}$ with a condition on $\dot{\rho}$, i.e. $\|\dot{\rho}\|_{2} \leq u=\gamma / \beta$ implies that $\left\|\dot{x}_{0}\right\|_{2} \leq \gamma$ by $(5)$.

It is clear from the bound on $\gamma$ that it is desirable to have $c_{2}$ as small as possible and $c_{3}$ as large as possible in order to allow for rapid variations in $x_{0}$. This will also maximize the region $E \backslash F$. Since $c_{2}$ and $c_{3}$ only enters as a function of the quotient $c_{3} / c_{2}$, the obvious approach is to try to find $P(\rho)$ and $c_{i}, \mathrm{i}=1,2,3$, that satisfies (10-11) and maximizes $c_{3} / c_{2}$. We will later on discuss how to do this in a systematic way.

\section{Relation to Previous Work}

The difference between the approach taken in this work as compared to what has previously been done is that typically it is assumed that there is a matrix $P(\rho)$, which satisfies (10) and

$$
P(\rho) A(\rho)+A^{T}(\rho) P(\rho) \leq-c_{3} I<0
$$

instead of (11), for which $\sum_{i=1}^{m}\left\|\frac{\partial P}{\partial \rho_{i}}(\rho)\right\|_{2} \leq c_{4} \forall \rho \in \Gamma$, e.g. [8, Chapter 5.7]. Notice that if $A(\rho)$ is Hurwitz uniformly in $\rho$, then by [8, Lemma 5.12] there is always a solution to this inequality $\forall \rho \in \Gamma$. Then the derivative of $V(z, \rho)$ along the trajectories of the system is given by

$$
\begin{aligned}
\dot{V}(z, \rho) & =z^{T} P(\rho)\left[g(z, \rho)-\dot{x}_{0}\right] \\
& +\left[g(z, \rho)-\dot{x}_{0}\right]^{T} P(\rho) z+z^{T} \dot{P}(\rho) z \\
& =z^{T}\left[P(\rho) A(\rho)+A^{T}(\rho) P(\rho)\right] z \\
& +z^{T} \dot{P}(\rho) z+2 z^{T} P(\rho)\left[h(z, \rho)-\dot{x}_{0}\right] \\
& \leq-c_{3}\|z\|_{2}^{2}+z^{T} \sum_{i=1}^{m} \frac{\partial P}{\partial \rho_{i}}(\rho) \dot{\rho}_{i} z \\
& +2 z^{T} P(\rho)\left[h(z, \rho)-\dot{x}_{0}\right] \\
& \leq-c_{3}\|z\|_{2}^{2}+c_{4} u\|z\|_{2}^{2}+2 c_{2} L\|z\|_{2}^{3}+2 c_{2} \gamma\left\|_{z}\right\|_{2} \\
& =\left[-c_{3}+c_{4} u+2 c_{2}\left(L\|z\|_{2}+\frac{\gamma}{\|z\|_{2}}\right)\right]\|z\|_{2}^{2}
\end{aligned}
$$

if $\|\dot{\rho}(t)\|_{\infty} \leq u, \forall t \geq 0, \forall \rho \in \Gamma$. The region $E \backslash F$ in which $\dot{V}(z, \rho)<0$ is now given by the set of $z$ such that $z \in D$ and

$$
-c_{3}+c_{4} u+2 c_{2}\left(L\|z\|_{2}+\frac{\gamma}{\|z\|_{2}}\right)<0
$$

which is equivalent to

$$
\left(\|z\|_{2}-\frac{c_{3}-c_{4} u}{4 c_{2} L}\right)^{2}-\frac{\left(c_{3}-c_{4} u\right)^{2}}{16 c_{2}^{2} L^{2}}+\frac{\gamma}{L}<0
$$

We first notice that there is a solution to this inequality if

$$
\begin{aligned}
\gamma & <\frac{c_{3} \beta}{c_{4}}+8 L\left(\frac{c_{2} \beta}{c_{4}}\right)^{2} \\
& -\sqrt{\left[\frac{c_{3} \beta}{c_{4}}+8 L\left(\frac{c_{2} \beta}{c_{4}}\right)^{2}\right]^{2}-\left(\frac{c_{3} \beta}{c_{4}}\right)^{2}}
\end{aligned}
$$

We notice that the bound is increasing in $c_{3} / c_{4}$ and decreasing in $c_{2} / c_{4}$. The sets $E$ and $F$ are given by

$$
\begin{aligned}
& E=\left\{z \mid\|z\|_{2} \leq \frac{c_{3}-c_{4} u}{4 c_{2} L}+\sqrt{\frac{\left(c_{3}-c_{4} u\right)^{2}}{16 c_{2}^{2} L^{2}}-\frac{\gamma}{L}}\right\} \cap D \\
& F=\left\{z \mid\|z\|_{2} \leq \frac{c_{3}-c_{4} u}{4 c_{2} L}-\sqrt{\frac{\left(c_{3}-c_{4} u\right)^{2}}{16 c_{2}^{2} L^{2}}-\frac{\gamma}{L}}\right\} \cap D
\end{aligned}
$$

If for both approaches the values of $c_{2}, c_{3}$ and $\gamma$ are the same, then we see that the region $E \backslash F$ is smaller because $c_{3}$ is modified by subtracting $c_{4} u$.

We conclude this section by noting that it is not so easy in this approach to choose the different values of $c_{2}, c_{3}$, and $c_{4}$. This is because we would simultaneously like to maximize $c_{3} / c_{2}$ and minimize $c_{4} / c_{2}$ in order to maximize the region $E \backslash F$ and maximize $c_{3} / c_{4}$ and minimize $c_{4} / c_{2}$ in order to maximize the bound for $\gamma$, but the optimization of these three quotients are not independent of one-another. We will see this more explicitly in an example later on.

\section{Lyapunov Functions for the LPV System}

In this section we will discuss how to find solutions to (1011). First we notice that we can normalize these inequalities with $c_{2}$ to obtain

$$
\begin{gathered}
0<\bar{c}_{1} I \leq \bar{P}(\rho) \leq I \\
\dot{\bar{P}}(\rho)+\bar{P}(\rho) A(\rho)+A^{T}(\rho) \bar{P}(\rho) \leq-\bar{c}_{3} I<0
\end{gathered}
$$

where $\bar{c}_{1}=c_{1} / c_{2}, \bar{c}_{3}=c_{3} / c_{2}$ and $\bar{P}(\rho)=P(\rho) / c_{3}$. Clearly we should just fix $\bar{c}_{1}$ to some small number. Then we should look for $\bar{c}_{3}=c_{3} / c_{2}$ and $\bar{P}(\rho)$ that satisfies the matrix inequalities and which maximizes $\bar{c}_{3}$. This will as already mentioned both maximize the bound on $\gamma$ and maximize the region $E \backslash F$. The optimization problem is however not tractable as it stands, since there are infinitely many 
constraints, since there are constraints for every value of $\rho \in \Gamma$. Several different approaches for how to circumvent this problem has been proposed in the literature, see e.g. $[3,6,1,7]$. One of the more appealing approaches, [7], assumes that $A(\rho)$ and $P(\rho)$ are rational functions of $\rho$ given in Linear Fractional Transformation (LFT) form. Then [7] show that the above optimization problem becomes a Semidefinite Program (SDP), which can be solved efficiently in polynomial time, e.g. [13] using very efficient interior-point algorithms.

The LPV system is not always such that the $A$-matrix is rational in $\rho$. However, any rational matrix-valued function can be realized as an LFT, e.g. [12]. On a compact domain $\Gamma$ any function can be arbitrarily well approximated by a rational function. Therefore for any $\varepsilon>0$ it will be possible to find an LFT-realization of a rational $A_{r}(\rho)$ such that $\|\tilde{A}(\rho)\|_{2} \leq \varepsilon, \forall \rho \in \Gamma$, where $\tilde{A}(\rho)=A(\rho)-A_{r}(\rho)$. This can be taken into account in the stability analysis in Section 3 by redefining (8), i.e. we write

$$
g(z, \rho)=A_{r}(\rho) z+h_{r}(z, \rho)
$$

where $h_{r}(z, \rho)=h(z, \rho)+\tilde{A}(\rho) z$. Then we do the rest of the analysis based on $A_{r}$ and $h_{r}$ instead of $A$ and $h$, where we make use of the fact that $h_{r}$ by (9) satisfies the bound

$$
\left\|h_{r}(z, \rho)\right\|_{2} \leq\|h(z, \rho)\|_{2}+\|\tilde{A}(\rho) z\|_{2} \leq L\|z\|_{2}^{2}+\varepsilon\|z\|_{2}
$$

It turns out that if $c_{3}$ in (11) is replaced with $c_{3}+2 c_{2} \varepsilon$ and $A(\rho)$ with $A_{r}(\rho)$, then the constraint on $\gamma$ and the region $E \backslash F$ will remain the same. We also have to add the constraint that $c_{3}>0$. Thus we have shown how to use an approximation $A_{r}$ of $A$ and obtain similar results as for rational $A$ assuming that we tighten the constraint in (11). Notice that after scaling with $c_{2}$ we will again have linear matrix inequalities, since the modification of $c_{3}$ is proportional to $c_{2}$. To summarize, the inequalities now read

$$
\begin{gathered}
0<\bar{c}_{1} I \leq \bar{P}(\rho) \leq I \\
\dot{\bar{P}}(\rho)+\bar{P}(\rho) A_{r}(\rho)+A_{r}^{T}(\rho) \bar{P}(\rho)+\bar{c}_{3} I \leq-2 \varepsilon I<0
\end{gathered}
$$

together with $\bar{c}_{3}>0$. Before concluding this section we remark that there are alternative ways of taking care of the approximation error $\tilde{A}$. One can consider an extended LFT description of $A_{r}$ by augmenting $\rho$ with entries that will take $\tilde{A}$ into account, e.g. [12]. This will increase the dimension of the LFT-description, and hence increase the computational complexity. Note that both approaches will result in a global Lyapunov function for the original LPV system. Therefore we do not believe it to be advantageous to use the latter approach.

\section{Example}

Let

$$
\begin{aligned}
& \dot{x}_{1}=\left(x_{1}-1\right) x_{1}+2(\rho+1) x_{2}+\frac{\rho^{2}}{400}\left(100-81 \rho^{2}\right) \\
& \dot{x}_{2}=2(\rho-1) x_{1}-x_{2}
\end{aligned}
$$

where $|\rho| \leq 0.5$ and $|\dot{\rho}| \leq u$. The Lipschitz constant may be taken as $L=2$. The linearized system becomes

$$
\begin{aligned}
& \dot{z}_{1}=\left(2 x_{10}-1\right) z_{1}+2(\rho+1) z_{2} \\
& \dot{z}_{2}=2(\rho-1) z_{1}-z_{2}
\end{aligned}
$$

where we will look at "frozen" stationary solutions given by $x_{10}=\rho^{2} / 20$ and $x_{20}=2(\rho-1) x_{10}$. Hence,

$$
\left\|\frac{\partial \varphi}{\partial \rho}\right\|_{2}=\frac{\rho}{10}\left\|\left[\begin{array}{c}
1 \\
3 \rho-2
\end{array}\right]\right\|_{2}=\frac{\rho}{10} \sqrt{9 \rho^{2}-12 \rho+5}
$$

The LPV system is given by

$$
\begin{aligned}
& \dot{z}_{1}=\left(\rho^{2} / 2-1\right) z_{1}+2(\rho+1) z_{2} \\
& \dot{z}_{2}=2(\rho-1) z_{1}-z_{2}
\end{aligned}
$$

which is stable for constant $\rho \in[-\sqrt{50 / 41}, \sqrt{50 / 41}]$. In the relevant interval $(\rho \in[-0.5,0.5])$, we have $\beta=$ $\max _{\rho}\left\|\frac{\partial \varphi}{\partial \rho}\right\|_{2}=0.1820$.

The $A$-matrix can be written as an LFT in $\rho$ :

$$
\begin{aligned}
A & =\left[\begin{array}{cc}
\frac{\rho^{2}}{10}-1 & 2(\rho+1) \\
2(\rho-1) & -1
\end{array}\right]=\left[\begin{array}{cc|c}
-1 & 2 \rho+2 & \frac{\rho}{10} \\
-2 & -1 & 2 \\
\hline 1 & 0 & 0
\end{array}\right] \star \rho \\
& =\left[\begin{array}{cc|cc}
-1 & 2 & 0 & 2 \\
-2 & -1 & 2 & 0 \\
\hline 1 & 0 & 0 & 0 \\
0 & 1 & \frac{1}{20} & 0
\end{array}\right] \star \underbrace{\left[\begin{array}{cc}
\rho & 0 \\
0 & \rho
\end{array}\right]}_{\Delta} \\
& =\left[\begin{array}{cc}
A_{0} & B_{0} \\
C_{0} & D_{0}
\end{array}\right] \star \Delta=A_{0}+B_{0}\left(I-\Delta D_{0}\right)^{-1} \Delta C_{0}
\end{aligned}
$$

where $\star$ denotes the Redheffer star product, e.g. [14]. We will here assume that the Lyapunov function is quadratic and parameterized by $V(x)=x^{T} P(\rho) x$, where

$$
P(\rho)=\left[\begin{array}{c}
I \\
\left(I-\Delta D_{0}\right)^{-1} \Delta C_{0}
\end{array}\right]^{T} P_{0}\left[\begin{array}{c}
I \\
\left(I-\Delta D_{0}\right)^{-1} \Delta C_{0}
\end{array}\right]
$$

Maximizing $\bar{c}_{3}$ subject to $10^{-9} I<P(\rho)<I$ and (15) for all $\rho \in[-0.5,0.5]$, yields $\bar{c}_{3}=1.0895$ and $\bar{c}_{4}=\max _{\rho}\left\|\frac{\partial P}{\partial \rho}\right\|_{2}=$ 0.957 . The stability region in terms of $c_{3}-u c_{4}$ with respect to $u$ is shown in Figure 1 as the dash-dotted line.

We can increase the stability region by maximizing $\bar{c}_{3}(u)-$ $u \bar{c}_{4}(u)$ for each value of $u$ subject to (15). The result is shown as the dashed line in Figure 1.

If we instead use (11) where $|\dot{\rho}| \leq u$ for maximizing $\bar{c}_{3}(u)$ we obtain an even larger stability region, see solid line in Figure 1. The stability regions are in this case defined by (13) and (14). The only difference compared to (16) and (17) is that $c_{3}-c_{4} u$ is substituted for $c_{3}(u)$. As can be seen in Figure 1, the stability region is extended in our approach compared to previous work. 


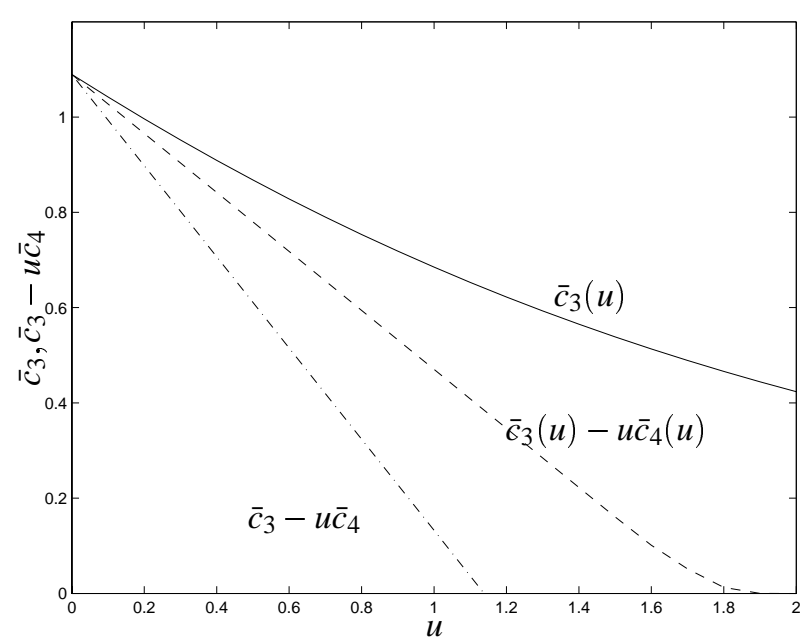

Figure 1: Stability region

If we let the rate of $\rho$ be bounded by $|\dot{\rho}| \leq u$, we get the stability regions as defined in (16) and (17). The limit on $\gamma$ according to (12) is 0.0317 which is obtained using (11) with $u=0.1744=\gamma / \beta$.

Using the related constants $\left(c_{3}=1.0079\right.$ and $\left.c_{2}=1\right)$ in (13) and (14), the sets $E$ and $F$ can be depicted in as in Figure 2.

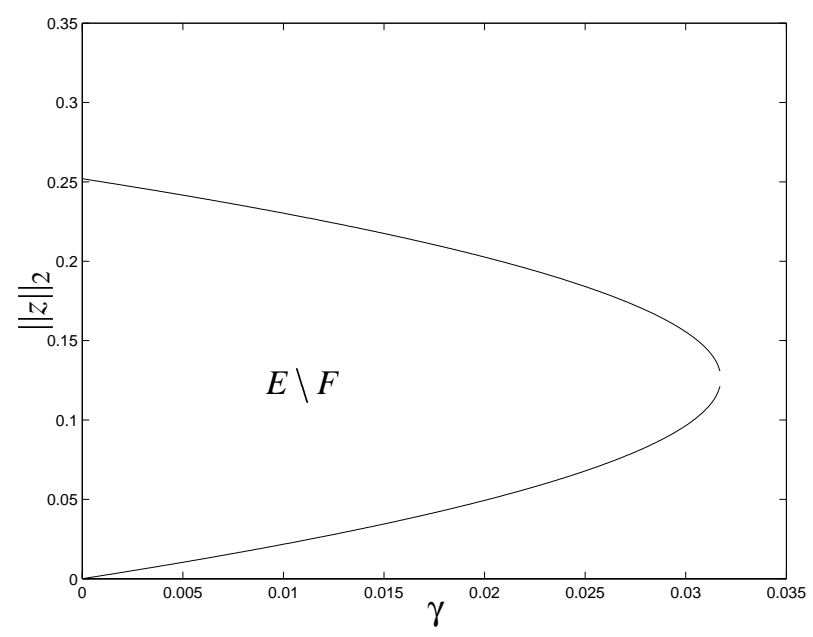

Figure 2: Convergence set $E \backslash F$ as a function of $\gamma$ for $c_{3}=1.0079$ and $c_{2}=1$.

\section{Conclusions}

In this paper we have analyzed stability of nonlinear systems for slowly varying parameters. We have shown that recent results for analysis of LPV systems can be used to obtain larger regions of stability for nonlinear systems as compared to previous approaches which only consider the linear time invariant system obtained for each fixed parameter value.
The framework presented admits extensions to consider robust models. It is also possible to take into account approximate descriptions of the function relating the parameter and the frozen state. This can be done by introducing bounds on the difference between the true system and a nominal system. This difference can therefore be treated similarly as the derivative of the nominal state.

\section{Acknowledgments}

The authors gratefully acknowledge financial support from the Swedish Research Council under contract No. 2712000-770.

\section{References}

[1] P. Apkarian and H. D. Tuan. Parameterized LMIs in control theory. SIAM J. Control and Optimization, 38(4):1241-1264, 2000.

[2] K. J. Åström and B. Wittenmark. Adaptive Control. Addison-Wesley, Reading, Massachusetts, 1995.

[3] S. Boyd, L. El Ghaoui, E. Feron, and V. Balakrishnan. Linear Matrix Inequalities in System and Control Theory. SIAM, Philadelphia, 1994.

[4] J. V. Breakwell, J.L. Speyer, and A. E. Bryson. Optimization and control of nonlinear systems using the second variation. SIAM J. Control A.1., page 193, 1963.

[5] A. E. Bryson and Y.-C. Ho. Applied Optimal Control. Ginn and Company, 1969.

[6] P. Gahinet, P. Apkarian, and M. Chilali. Affine paremeter-dependent Lyapunov functions and real parameteric uncertainty. IEEE Transactions on Automatic Control, 41(3):437-442, 1996.

[7] T. Iwasaki and G. Shibata. LPV systemanalysis using quadratic separator for uncertain implicit systems. IEEE Transactions on Automatic Control, AC-46:11951208, 2001.

[8] H. K. Khalil. Nonlinear Systems. Prentice Hall, Upper Saddle River, 1996.

[9] H. K. Khalil and P. V. Kokotovic. On stability properties of nonlinear systems with slowly varying inputs. IEEE Transactions on Automatic Control, AC-36:229, 1991.

[10] D. A. Lawrence and W. J. Rugh. On a stability theorem for nonlinear systems with slowly varying inputs. IEEE Transactions on Automatic Control, AC-35:860-864, 1990.

[11] A. M. Lyapunov. The general problem of the stability of motion (translated into English by A. T. Fuller). International Journal of Control, 55:531-773, 1992.

[12] J. F. Magni. Linear fractional representations with a toolbox for use with MATLAB. Technical Report TR 240/01 DCSD, Dept. of Systems Control and Flight Dynamic, ONERA, 2001. 
[13] Y. Nesterov and A. Nemirovsky. Interior-Point Polynomial Methods in Convex Programming. SIAM, Philadelphia, 1994.

[14] R. Redheffer. Inequalities for a matrix Riccati equation. Journal of Mathematics and Mechanics, 8, 1959.

[15] W. J. Rugh. Analytical framework for gain scheduling. IEEE Control Systems Magazine, 11(1):79-89, 1991.

[16] J. S. Shamma and M. Athans. Anaysis of gain scheduled control for nonlinear plants. IEEE Transactions on Automatic Control, AC-35(8):898-907, 1990.

[17] J. S. Shamma and M. Athans. Guaranteed properties of gain scheduled control for linear parameter-varying plants. Automatica, 27(3):559-564, 1991.

[18] J. S. Shamma and M. Athans. Gain scheduling: Potential hazards and possible remedies. IEEE Control Systems Magazine, 12(3):101-107, 1992.

[19] G. Stein. Adaptive flight control: A pragmatic view. In K. S. Narendra and R. V. Monopoli, editors, Applications of Adaptive Control. Academic Press, New York, 1980. 